\title{
3D Numerical Simulations of $f$-Mode Propagation Through Magnetic Flux Tubes
}

\author{
K. Daiffallah ${ }^{1}$. T. Abdelatif ${ }^{1}$ - A. Bendib ${ }^{2}$. \\ R. Cameron $^{3}$. L. Gizon ${ }^{3}$
}

(C) Springer $\bullet \bullet \bullet$

\begin{abstract}
Three-dimensional numerical simulations have been used to study the scattering of a surface-gravity wave packet by vertical magnetic flux tubes, with radii from $200 \mathrm{~km}$ to $3 \mathrm{Mm}$, embedded in stratified polytropic atmosphere. The scattered wave was found to consist primarily of $m=0$ (axisymmetric) and $m=1$ modes. It was found that the ratio of the amplitude of these two modes is strongly dependant on the radius of the flux tube: The kink mode is the dominant mode excited in tubes with a small radius while the sausage mode is dominant for large tubes. Simulations of this type provide a simple, efficient and robust way to start understanding the seismic signature of flux tubes, which have recently began to be observed.
\end{abstract}

Keywords: Helioseismology, Direct Modeling; Waves, Magnetohydrodynamic; Magnetic Fields

\section{Introduction}

Magnetic flux tubes couple the different layers of the solar atmosphere, and waves propagating along the magnetic-field lines are a possible source of the mechanical-energy transport required to heat the chromosphere and corona. The waves also provide a helioseismic means of constraining the properties of the magnetic tubes which is complementary to observational studies with SOHO/MDI data (Duvall. Gizon, and Birch, 2006) and Hinode (Fuijmura and Tsuneta, 2009). In the latter work the authors found that the observed Poynting flux is substantial when compared to the flux required to heat the chromosphere and corona. Unfortunately with observations at a single height they were unable to identify what mixture of $m=0$, axisymmetric sausage-modes, and $m=1$, kinkmodes, they were observing. Theoretical studies of the interaction of flux tubes

\footnotetext{
1 Observatory of Algiers, CRAAG, Algiers, Algeria, email: k.daiffallah@craag.dz

2 University of Sciences and Technology (USTHB), Faculty of Physics, Algiers, Algeria

3 Max-Planck-Institut für Sonnensystemforschung,

Katlenburg-Lindau, Germany
} 
and waves have been performed in the past, for example Roberts and Webb (1979), Wilson (1980), Spruit (1981), Spruit (1982), Bogdan and Knölker (1989), Solanki (1993), Bogdan et al. (1996), Hasan and Kalkofen (1999), Tirrv (2000), Gizon. Hanasoge. and Birch (2006), Jain and Gordovskyv (2008), Hanasoge et al. (2008), Hanasoge and Cally (2009). It is now well established that slender magnetic flux tubes permit the propagation of the two basic types of magnetohydrodynamic waves: The longitudinal tube waves (sausage modes) with azimuthal wave number $m=0$, which are axisymmetric, excited by pressure fluctuations. The transversal tube waves (kink modes) with $m= \pm 1$, which are nonaxisymmetric and describe the incompressible undulations of the tube, supported by magnetic tension. However, in these various papers, simplifying assumptions were used, such as the thin-flux-tube approximation, isothermal or unstratified atmospheres, and the neglect of higher- $m$ modes. Hanasoge et al. (2008) included a stratified atmosphere and used the thin-flux-tube approximation to evaluate the scattering matrix associated with an $f$-mode wave interacting with a flux tube. The thin-flux-tube approximation works when the radius of the tube is smaller than it scale height which was $260 \mathrm{~km}$. For this reason the largest tube that they considered was $160 \mathrm{~km}$. Numerical treatment of flux tubes of arbitrary size is straightforward and robust. The approach is already yielding interesting results in the context of sunspots, see e.g. Cameron. Gizon. and Daiffallah (2007), Hanasoge (2008), Khomenko, Collados, and Fellipe (2008), Cameron, Gizon, and Duvall (2008), Khomenko et al. (2009).

In this paper we use numerical simulations to explore the response of vertical, small scale flux-tubes with radii between $200 \mathrm{~km}$ and $3 \mathrm{Mm}$. The paper is structured as follows: In Section 2 we present the background model and the equations describing the wave propagation. In Section 3 we present the results of a series of convergence tests. These are essential since we are considering tubes with small radii. In Section 4 we study the scattered wave field, Section 5 is devoted to mode conversion, before discussing the results in Section 6 .

\section{Simulation Model}

We use a slightly modified version of the SLiM (Semi spectral Linear MHD) code described in Cameron. Gizon. and Daiffallah (2007) to propagate linear waves through an enhanced polytropic atmosphere. The enhanced polytropic atmosphere we use was constructed by following Cally and Bogdan (1997). Specifically we follow those authors in considering a $4820 \mathrm{G}$ purely vertical flux tube along the axis of which the pressure, density, and temperature vary as a polytrope. The polytropic index is set as 1.5 , the density at a depth of $1 \mathrm{Mm}$ is set to $10^{-5} \mathrm{~g} \mathrm{~cm}^{-3}$. The sound speed is set to be equal to the Alfven speed at depth of $400 \mathrm{~km}$. The external atmosphere is the enhanced polytrope obtained by requiring that the total (gas and magnetic) pressure be horizontal uniform, and by that the density is that of the original polytrope. Since the magnetic pressure is independent of height this means that away from the flux tube the pressure is increased by a comstant which is independent of height and thus does not affect the vertical hydrostatic force balance. We emphasise that this is the 
same formulation as was used by Cally and Bogdan (1997) and is a reasonable approximation for qualitatively studying wave propagation in the upper part of the convection zone. Figure 1 show a comparison between the vertical profiles of the sound speed, pressure, and acoustic velocity for the standard solar model $\mathrm{S}$ by Christensen-Dalsgaard and those on the flux-tube axis. We note that with our boundary conditions waves are strongly reflected from the upper boundary which restricts us to considering waves below the acoustic cut-off frequency. As in Cally and Bogdan (1997), we restrict ourselves to purely vertical flux tubes. In our case this is partly motivated by the fact that we are considering only small tubes (not sunspots) which are expected to be almost vertical until they expand into the overlying atmosphere. We thus are considering relatively small flux tubes of different sizes and are including the full three-dimensional geometry.

Our code solves the linearized, ideal MHD equations in three dimensions. We write the magnetic, velocity, pressure, and density perturbation in terms of the displacement vector $[\boldsymbol{\xi}]$. The background is assumed to be time independent. The primed quantities in the equations are the Eulerian perturbation, whereas the background variables are subscripted with 0 . The gravitational acceleration $[\boldsymbol{g}]$ is constant, and there is no background steady flow.

The equation governing the displacement vector is:

$$
\rho_{0} \frac{\partial^{2} \boldsymbol{\xi}}{\partial t^{2}}=-\nabla p^{\prime}+\rho^{\prime} \boldsymbol{g}+\frac{1}{4 \pi}\left(\mathbf{j}^{\prime} \times \mathbf{B}_{0}+\mathbf{j}_{0} \times \mathbf{B}^{\prime}\right)
$$

The perturbed quatities: density $[\rho]$, pressure $[p]$, magnetic field $[\mathbf{B}]$. Electric current $[\mathbf{j}]$ are written as functions of $[\boldsymbol{\xi}]$ as follow:

$$
\begin{gathered}
\rho^{\prime}=-\nabla \cdot\left(\rho_{0} \boldsymbol{\xi}\right), \\
p^{\prime}=c_{0}^{2}\left(\rho^{\prime}+\boldsymbol{\xi} \cdot \nabla \rho_{0}\right)-\boldsymbol{\xi} \cdot \nabla p_{0}, \\
\mathbf{B}^{\prime}=\nabla \times\left(\boldsymbol{\xi} \times \mathbf{B}_{0}\right), \\
\mathbf{j}^{\prime}=\nabla \times \mathbf{B}^{\prime},
\end{gathered}
$$

A flux tube that is almost evacuated at the surface and that has a radial profile is superposed on the background atmosphere. The magnetic flux tubes have a top-hat profile given by $B(r)=B_{0} \exp \left(-r^{4} / r_{0}^{4}\right)$ where $r_{0}$ is the tubes radius, and $B_{0}=4820 \mathrm{G}$ (Cally and Bogdan, 1997). We use a local Cartesian geometry defined by the horizontal coordinates $x$ and $y$. The vertical coordinate is $z$, and the simulation covers the height range from $0.2 \mathrm{Mm}$ to $6 \mathrm{Mm}$ below the solar surface. Note that the convention adopted here is that $z$ incresaes with depth. The horizontal extent of the domain is $x \in[-20,20] \mathrm{Mm}$ and $y \in[-10,10]$ $\mathrm{Mm}$. The axis of the vertical flux tube passes through the point $x=-7 \mathrm{Mm}, y$ $=0$. 

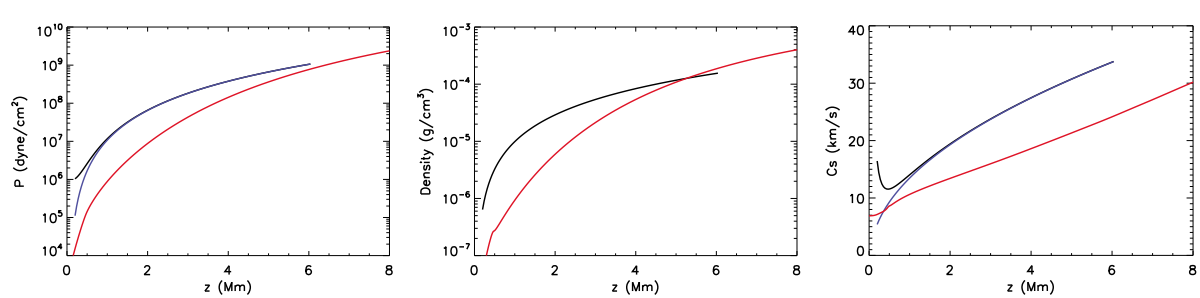

Figure 1. The vertical profiles of the pressure, density and acoustic velocity for the background model. Red plots are the profiles for the standard solar model S by ChristensenDalsgaard (with $z=0$ corresponding to the height of $400 \mathrm{~km}$ in model S). Blue plots are for the polytrope along the flux-tube axis, and the black plots are for the enhanced polytrope in quiet the Sun.

At $t_{0}=0$, an $f$-mode wave packet with a Gaussian profile in Fourier space is situated at $x_{0}=-20 \mathrm{Mm}$ and it propagates from left to right in the $x$-direction.

Specifically the initial condition is constructed from individual $f$-modes each of which has the usual (wavenumber-dependent) exponential dependence on height. The phase of the each eigenmode is set so that the waves are all in phase at $x=-20 \mathrm{Mm}$. The distance from the initial condition and the sunspot was chosen as the result of a compromise: On the one hand it is desirable for the initial wavepacket to be close to the sunspot (so that the wave attenuation before the wave reaches the sunspot is minimized), on the other hand there is a relationship between the wavepacket and cross covariance from random sources (Cameron, Gizon, and Duvall, 2008; Gizon, Birch, and Spruit, 2010) which only applies outside the "near field" of the initial condition. The frequency of each mode is $\sqrt{g k}$ where $k$ independently of the details of the background quiet-Sun model. The vertical component of the velocity, $\partial_{t} \xi_{z}$, at the upper surface is our main diagnostic, as it is closely related to the observable Doppler velocity at disk centre. The amplitude of the different modes in the initial condition depends on their eigenfrequency. The amplitudes of the surface velocity is a Gaussian with a central frequency and the half width of the wave packet are $3 \mathrm{mHz}$ and 1.18 $\mathrm{mHz}$ respectively.

The top boundary also corresponds to that of Cally and Bogdan (1997) and has also been previously described in Cameron. Gizon, and Duvall (2008). It corresponds to a free surface in the non-magnetic regions and hence is effective in reflecting waves. This means that the effective acoustic cut-off of the model is infinite. Our initial condition has very little energy ( $\approx 0.06 \%$ above $5.3 \mathrm{mHz}$ ).

We have also performed a simulation without the flux tube being present, that is with the enhanced polytrope being used everywhere and with $B_{0}=0$. This simulation acts as a reference and allows us to construct the scattered wave field as the difference between the simulations with and without the flux tube.

An example of the wave interacting with a tube with a radius of $500 \mathrm{~km}$ is shown in Figure 2. For a tube of this size, the scattered wave is almost circular, with a significant amount of back scattering. The SLiM code assumes that the box is periodic, which accounts for the wave fronts coming in from the side of the box after 4000 seconds. It is to be noted that the diameter of the tube is neither small nor large with respect to the wavelength of the incoming waves, so that various types of tube modes will be excited. 

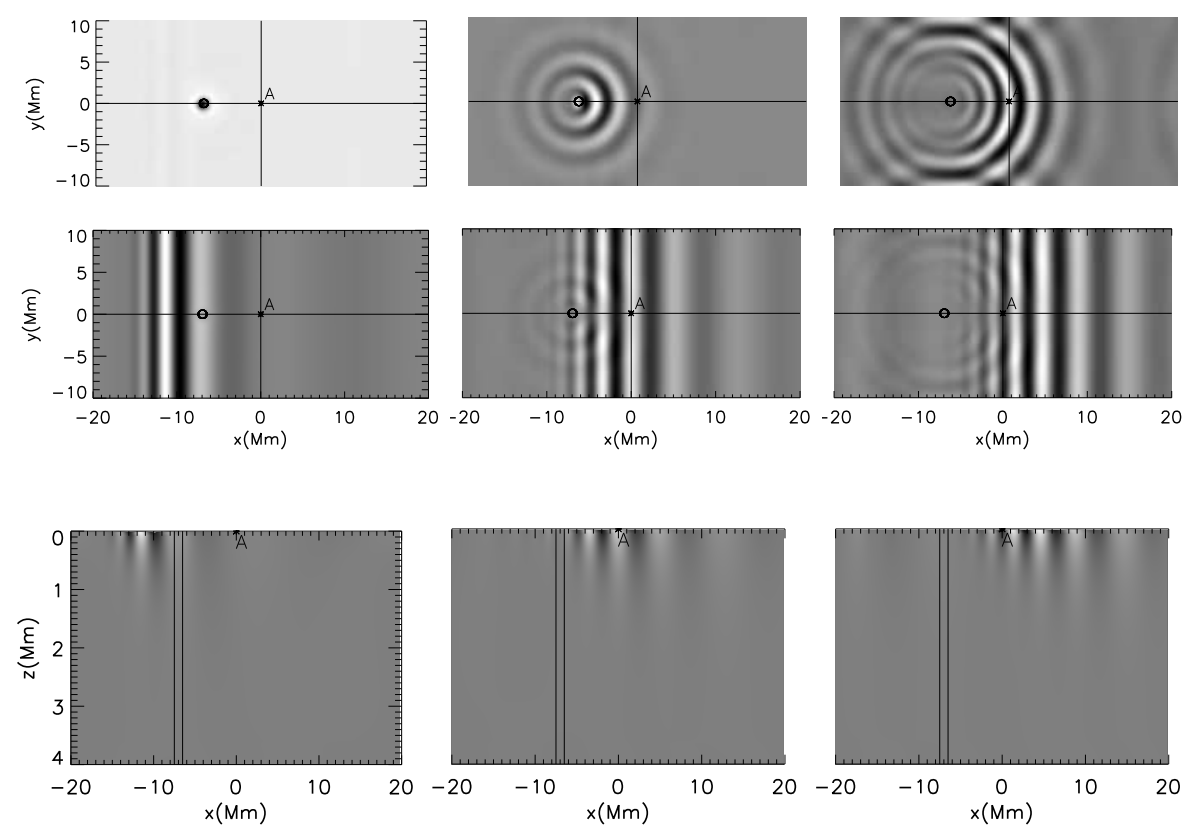

Figure 2. Wave propagation at three times (1600, 3000, and 4000 seconds after the start of the simulation), in the presence of a $500 \mathrm{~km}$ magnetic flux tube. The top panel shows the scattered component of the wave field near the "solar surface", the middle panel shows the full wave field whilst the lower panel is a vertical cut. The location and size of the tube is indicated by the circle at $x=-7 \mathrm{Mm}, y=0$. The point "A" is the location at which the resolution test analysis was performed (Section 3). Notice that the simulation is periodic in the horizontal directions, which can be seen in the scatterd wave field at later times.

\section{Resolution Test}

Our simulation box has a length of $40 \mathrm{Mm}$ in the direction in which the wave is propagating. The radii $\left[r_{o}\right]$ of the flux tubes we are interested in vary from $200 \mathrm{~km}$ to $3 \mathrm{Mm}$. Furthermore, since we have used a top-hat profile $[B(r)=$ $\left.B_{0} \exp \left(-r^{4} / r_{0}^{4}\right)\right]$ the "sidewalls" of the tube are even thinner. This raises the question of how many Fourier modes are required in the simulation to resolve the flux tubes. In the vertical direction we have found that 200 grid points are sufficient for all the flux tubes, probably because the flux tubes have no special structure in the $z$-direction. The tubes radii we have considered are $200 \mathrm{~km}$, $500 \mathrm{~km}, 1 \mathrm{Mm}$, and $3 \mathrm{Mm}$. We have considered three different choices for the number of horizontal Fourier modes: $50 \times 25,100 \times 50,200 \times 100$. The data of the scattered wave field at the point " $\mathrm{A}$ " is shown as a function of time in the top four panels of Figure 3, The three different resolutions are shown using different colors. It is apparent that the scattered wave at " $\mathrm{A}$ " is resolved for $r_{0}$ equal to $1 \mathrm{Mm}$ and $3 \mathrm{Mm}$ for all three choices of the number of Fourier modes. The degree of convergence in the $r_{0}=500 \mathrm{~km}$ case is reasonable, especially for the two higher resolutions. The case where $r_{0}=200 \mathrm{~km}$ is clearly a long way from convergence for at least the two lower resolutions. At the lowest resolution, the flux tube is significantly smaller than $40 \mathrm{Mm} / 50$ modes $=800 \mathrm{~km}$, for this reason 
the simulated waves apparently do not even see the tube and the scattered wave is absent. With 100 modes, the simulation appears more reasonable but the amplitude is only about $1 / 2$ of that of the simulation with 200 modes. The results using 200 modes should be much closer to the converged value: The relative resolution is similar to the case where we used 100 modes for the 500 $\mathrm{km}$ tube. The four lower panels of Figure 3 show the scattered wave along the line $y=0$ as a function of $x$ at a fixed time. The convergence properties are the same as we found for fixed position "A". To quantify the errors we introduce a measure for the difference between the simulations. Again concentrating on the point "A", we compare the time series of the vertical velocity from either the low or medium-resolution simulations $\left[v_{z}\right]$ with the high resolution simulation $\left[v_{z}^{h}\right]$ :

$$
\epsilon=\frac{\sum_{t}\left[v_{z}(t)-v_{z}^{h}(t)\right]^{2}}{\sum_{t} v_{z}^{h}(t)^{2}},
$$

We can also construct a measure of the error based upon the cuts at fixed time (i.e. the lower four panels of Figure 3). The error in this case is taken to be

$$
\epsilon=\frac{\sum_{x}\left[v_{z}(x)-v_{z}^{h}(x)\right]^{2}}{\sum_{x} v_{z}^{h}(x)^{2}}
$$

Figure 4 shows these measures of the error in various ways. In the left panels we see that the error decreases rapidly as the tube increases in radius. The panels on the right show that increasing the resolution by a factor of two decreases this measure of the error by a factor of about ten. This would suggest that the error associated with the highest resolution (200 modes) when applied to the smallest flux tube (200 km radius) should be approximately $3 \%$ (i.e. a factor of ten better than the simulation with 100 modes which has a $30 \%$ error).

\section{Scattering of Tubes}

The dependence of the magnitude of the scattering on radius can be seen in Figure 5 where the full and the scattered wave field are shown for tubes with radii $200 \mathrm{~km}, 500 \mathrm{~km}, 1 \mathrm{Mm}$, and $3 \mathrm{Mm}$. A vertical cut through the spot, along the $x$-axis, is shown in Figure [6] for tubes with radii $200 \mathrm{~km}, 1 \mathrm{Mm}$, and $3 \mathrm{Mm}$. For the two larger tubes, some mode conversion is apparent.

Naturally the simulations give us the full vector-velocity field and hence we can look simultaneously at all three components of the velocity field as shown in Figure 7. This figure makes it clear that the scattered field consists mainly of different mixtures of $m=0$ and $m=1$ modes for different radii. From this figure we can see that tubes with radius between $500 \mathrm{~km}$ and $2 \mathrm{Mm}$ are excited primarily in the $m=0$ mode, the upper part of the cylinder moving inward or outward (sausage modes). For the $z$-component of velocity, tubes with radii less than $500 \mathrm{~km}$ are excited with $m= \pm 1$ dipole oscillation whereas tubes between $500 \mathrm{~km}$ and $2 \mathrm{Mm}$ oscillate with a mixture of $m=0$ and $m=1$ modes. It is 

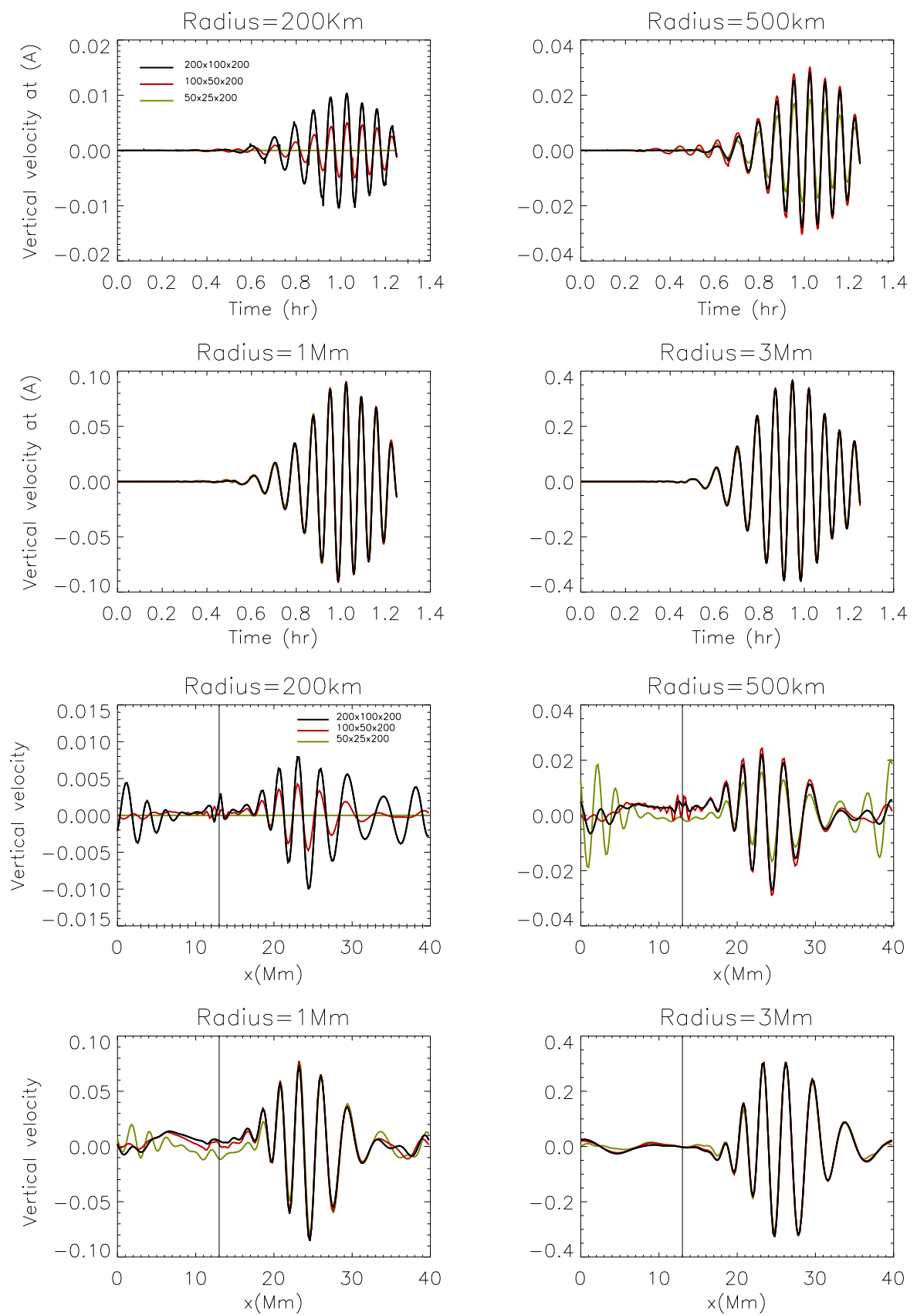

Figure 3. Comparison of results at different resolutions for various sizes flux tubes. The top four panels show the scattered component of the wave at a fixed point ("A"in Figure 2) as a function of time. The lower four panels show the scattered wave component at different locations along the $x$-axis at fixed time (corresponding to the middle panels in Figure 2 ) 

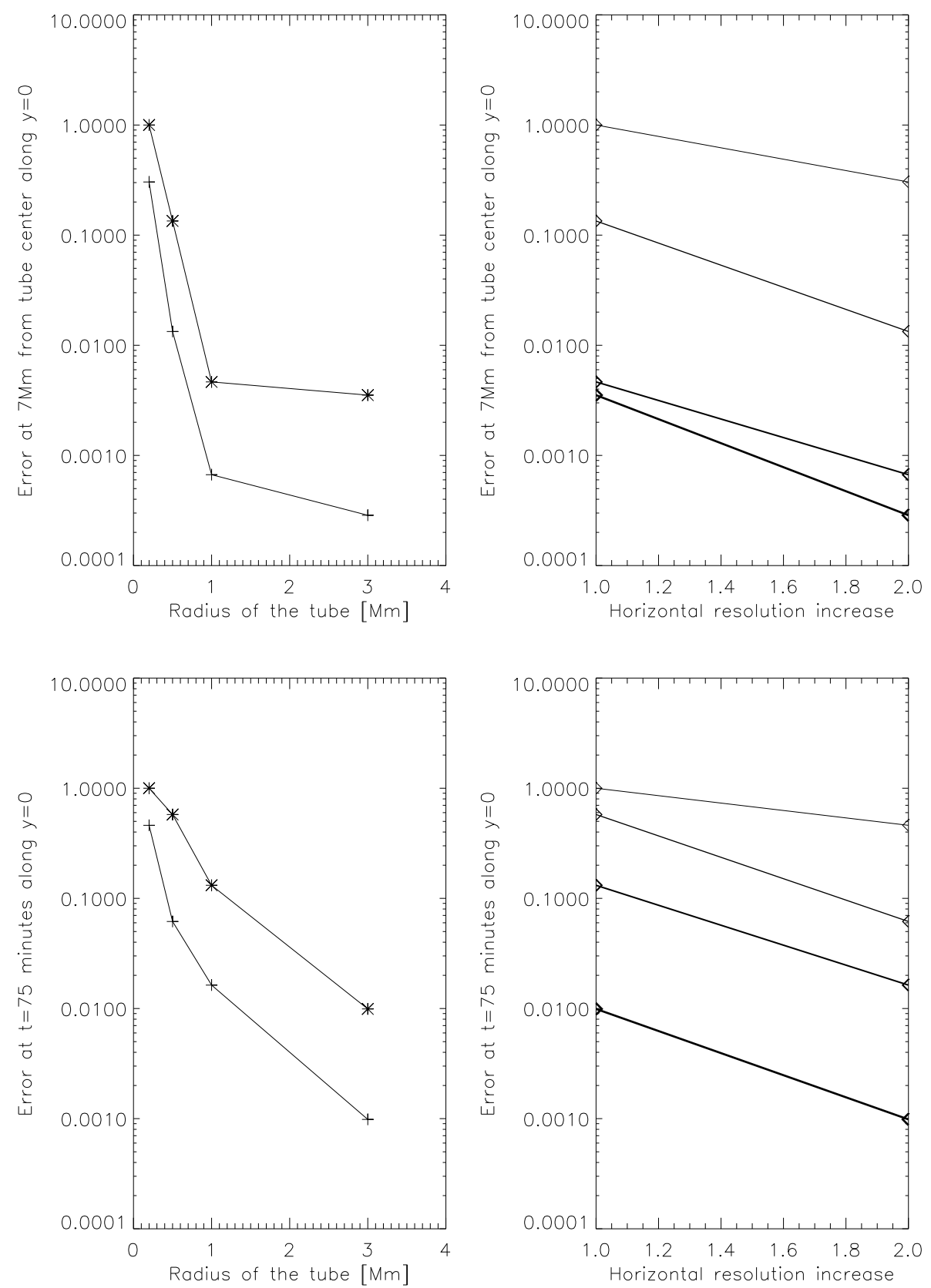

Figure 4. The error function for scattered wave field of vertical velocity, top panel at $7 \mathrm{Mm}$ from tube center along $y=0$, bottom panel at time $=75$ minutes along $y=0$. Curve with stars at left correspond to the difference low vs high resolutions, curve with cross is for the difference medium vs high resolutions. The right curves shows the variation of the error for the different radius when the horizontal resolution increases. 

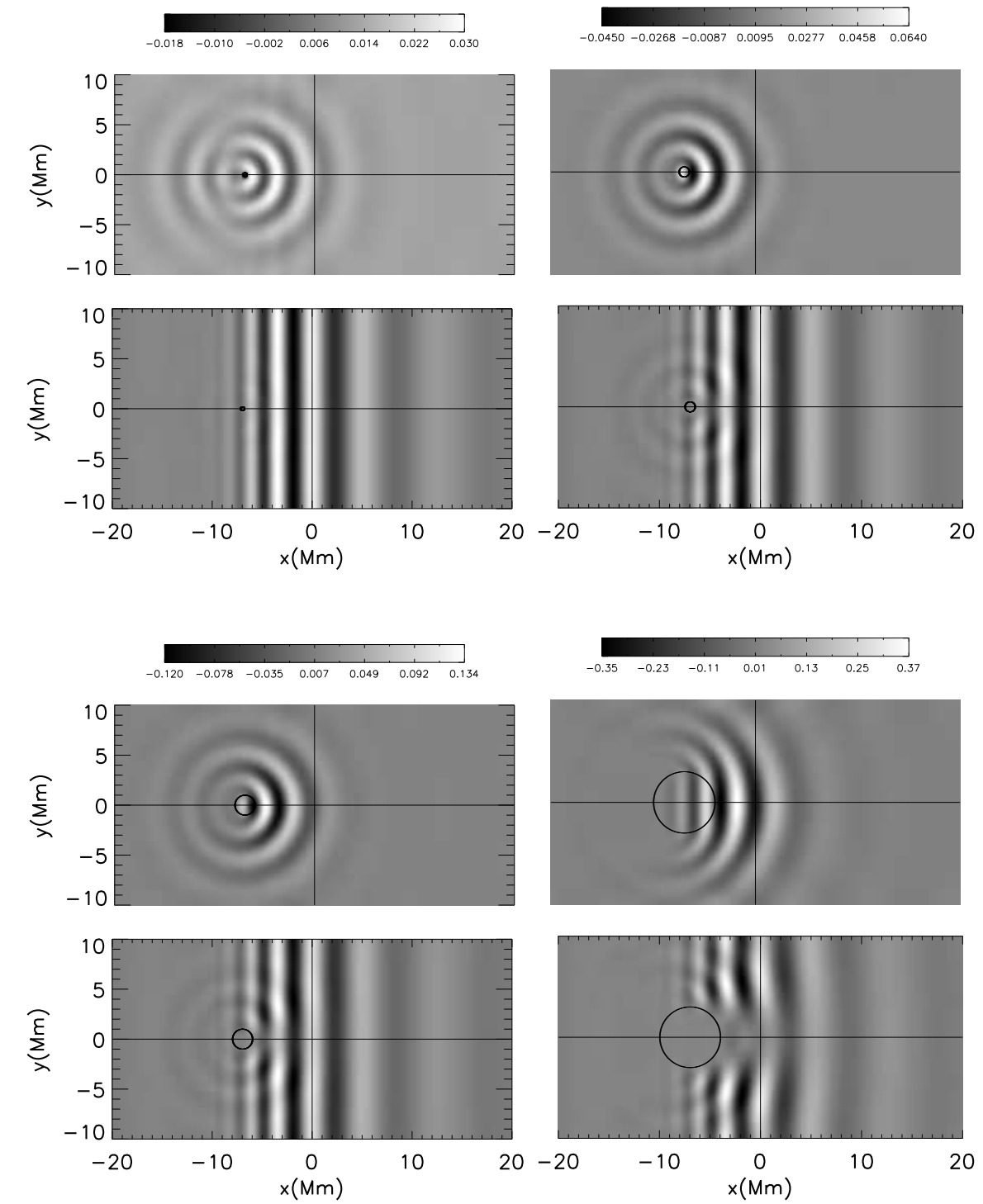

Figure 5. Wave propagation through tubes of different sizes. The images are from snapshots taken 3000 seconds after the start of the simulation. The upper four panels show vertical velocity scattered wave field (at the top) and the full wave field (at the bottom) for tubes with radii $200 \mathrm{~km}$ and $500 \mathrm{~km}$, the lower four panels are for tubes with radii $1 \mathrm{Mm}$ and $3 \mathrm{Mm}$. The circles indicate the sizes of these tubes. The simulation is periodic in the horizontal directions which can be seen in the scattered wave field. 

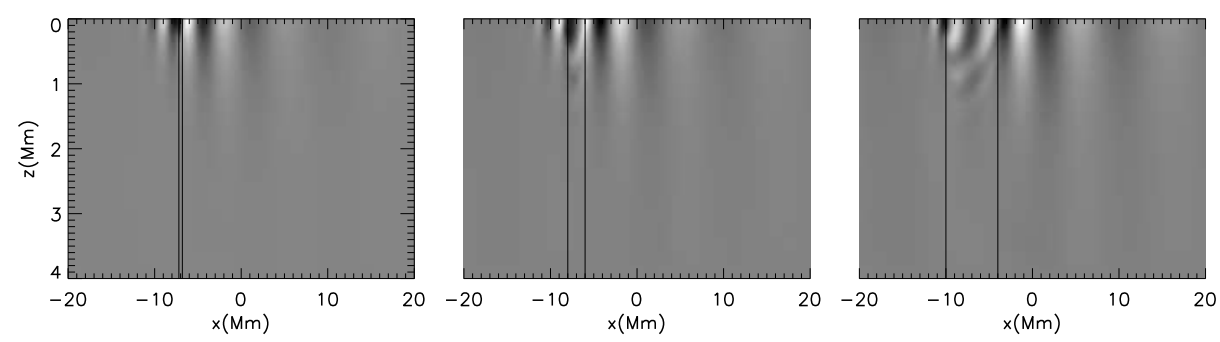

Figure 6. The $x$-component of the velocity near the photosphere; a vertical cut passing through the centre of the tubes $200 \mathrm{~km}, 1 \mathrm{Mm}, 3 \mathrm{Mm}$. All panels are from a snapshot at $t=$ 2000 seconds.

$\left(V_{x}\right)$
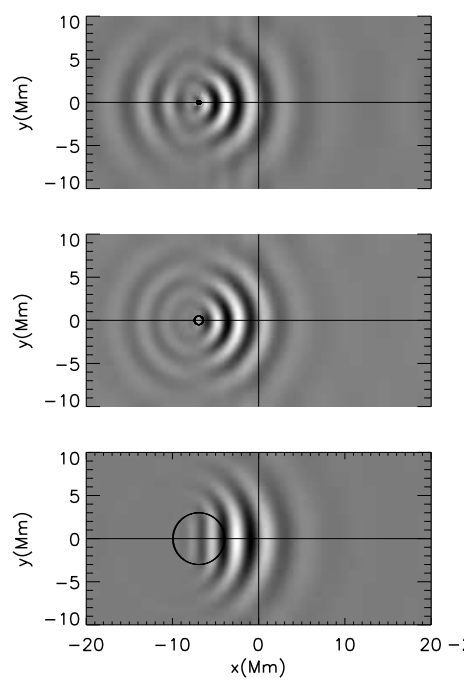

$\left(V_{y}\right)$
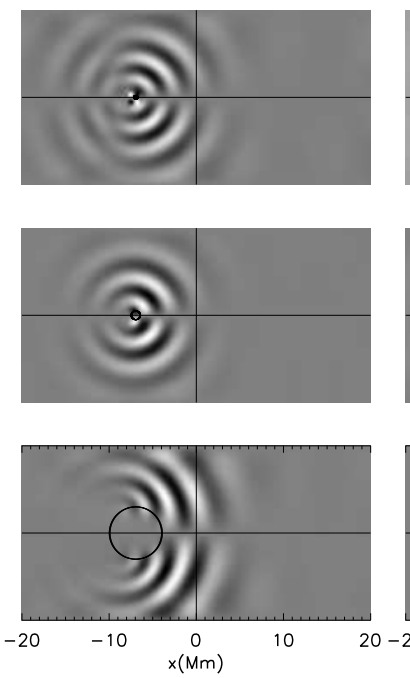

$\left(V_{z}\right)$
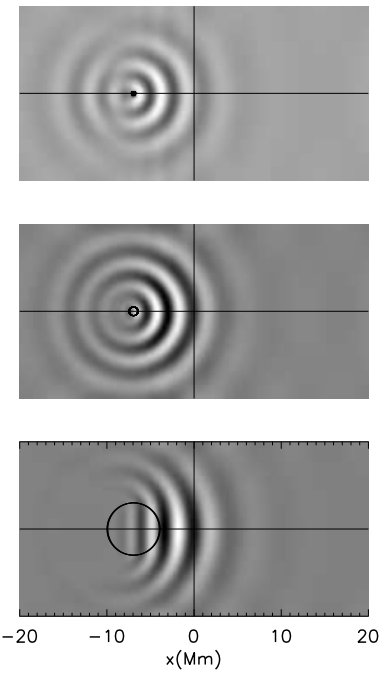

Figure 7. Scattered wave field for different tubes radii taken 3000 seconds after the start of the simulation. Radius from top to bottom: $200 \mathrm{~km}, 500 \mathrm{~km}, 3 \mathrm{Mm}$. Velocity component from left to right: $V_{x}, V_{y}, V_{z}$. Note that different gray-scales are used for each frame so that only the structure of the scattered wavefield is significant

more difficult to identify the oscillation of $3 \mathrm{Mm}$ tube radius, which presumably has substantial energy in higher $m$ components.

To procede further we consider the displacement of the tube with respect to its surroundings. The idea is illustrated in Figure 8. Thinner flux tubes (radius smaller than $500 \mathrm{~km}$ ) are effectively coupled to the motion of the surrounding plasma by the drag force, while larger tubes (radius larger than $500 \mathrm{~km}$ ) can move relative to the surrounding plasma owing to the action of the magnetic force, similar to a flexible solid body immersed in a fluid (Solanki, Inhester, and Schüssler, 2006). This result was expected, but is still nice to see in the simulations.

To better understand the $m$ modes that have been excited in the tube, we look at the total $x$-displacement not only along axis of the tube but also near its 

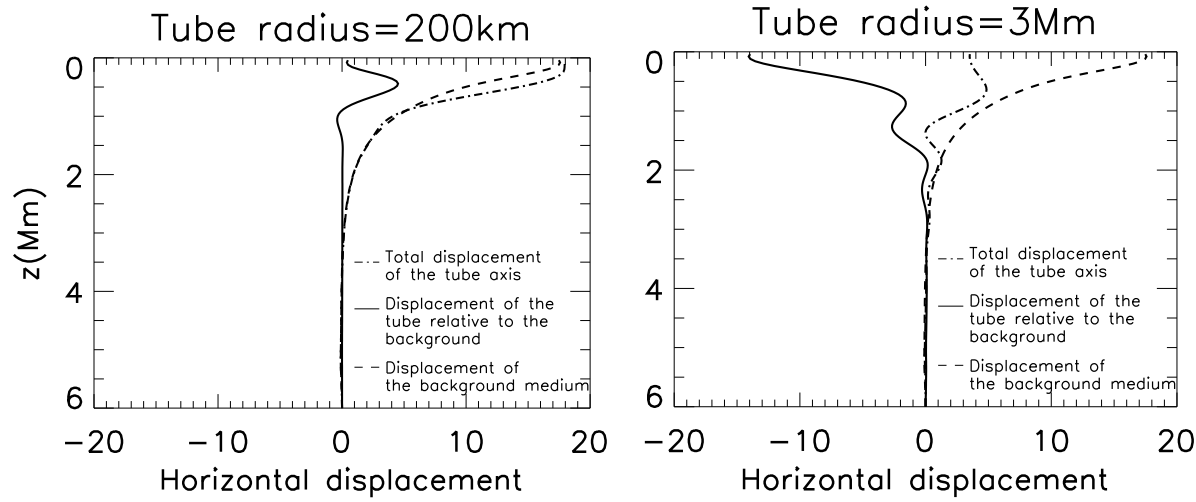

Figure 8. Horizontal displacement in arbitrary units in the $x$-direction of the tube axis and the background as a function of the depth $[z]$. The curves are taken 2000 seconds after the start of the simulation for $200 \mathrm{~km}$ tube radius, left, and $3 \mathrm{Mm}$ tube radius, right.
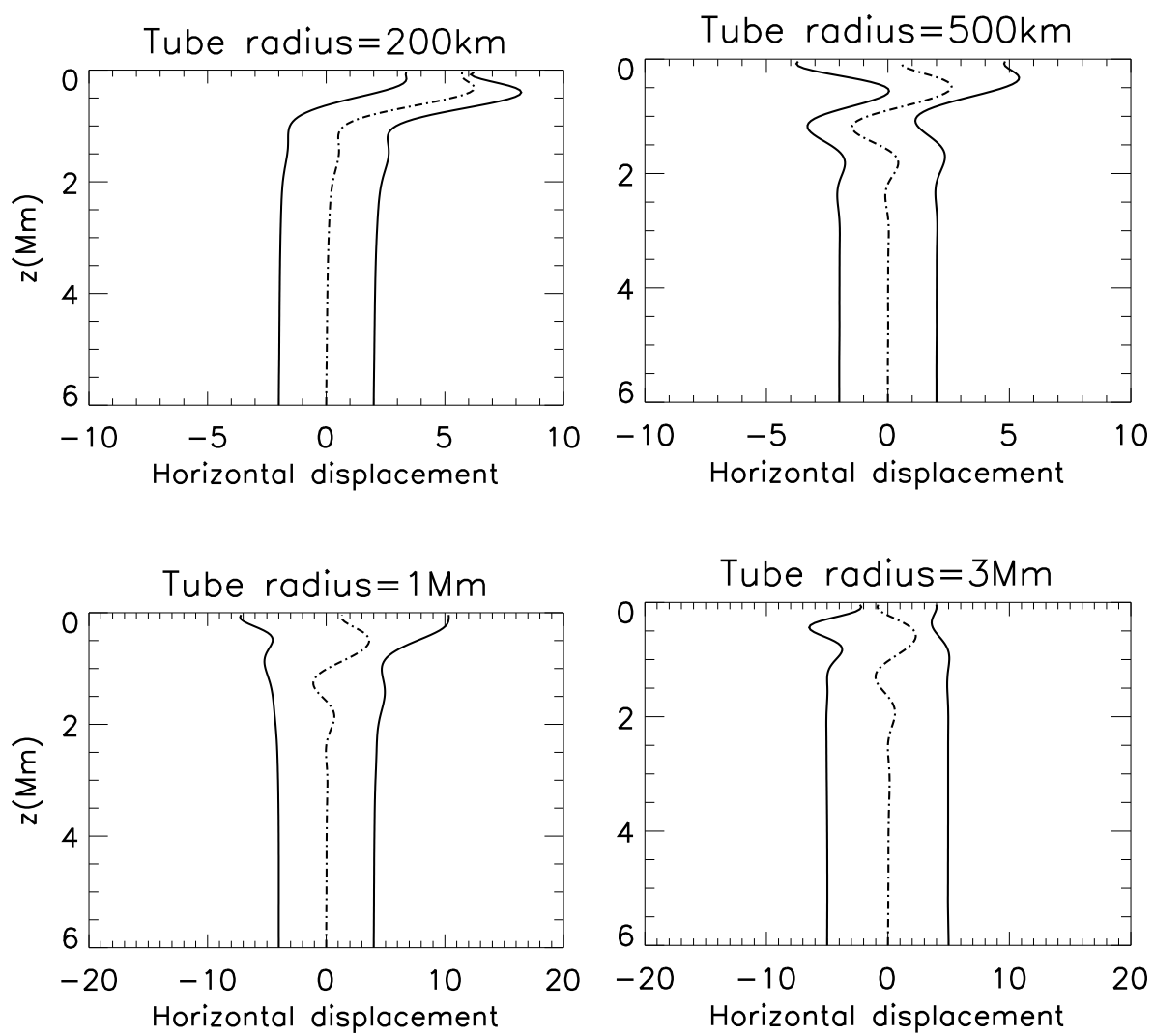

Figure 9. Horizontal displacement in arbitrary units in the $x$-direction of the tube axis (dashed line at the center) and the tube cross sections (full line right and left) as a function of the depth $[z]$. The curves are taken 1600 seconds after the start of the simulation for different tubes radii: $200 \mathrm{~km}, 500 \mathrm{~km}, 1 \mathrm{Mm}$, and $3 \mathrm{Mm}$ respectively. 

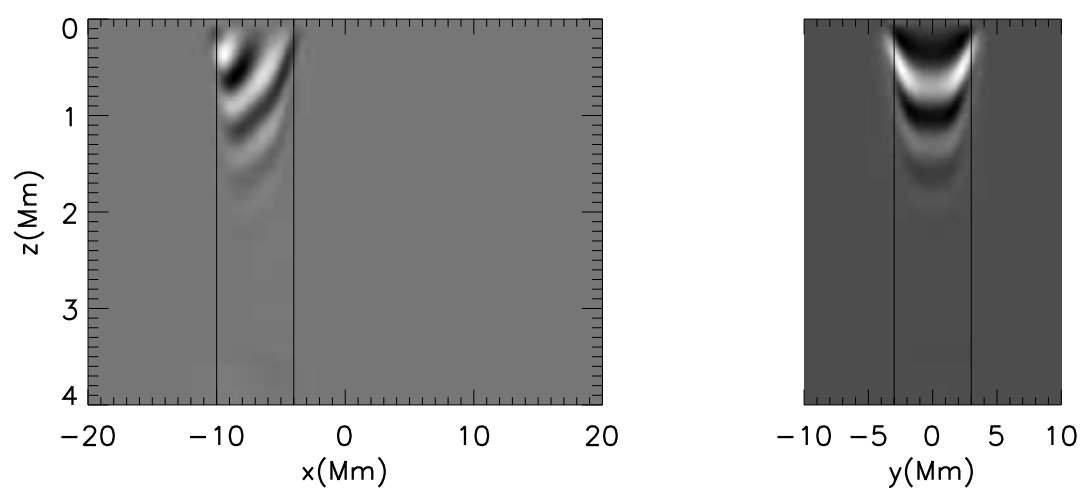

Figure 10. The $x$-component of the perturbation of the magnetic field introduced by the passage of the wave, shown in the $x-z$ (left) and $y-z$ (right) planes passing through the axis of the sunspot. The downward-propagating oscillations correspond to the slow-acoustic mode propagating along the fieldlines. This figure is for $t=2280$ seconds and the $3 \mathrm{Mm}$ flux tube.

edges. This is shown in Figure 9. Consistent with the above description, we see that the perturbations are coherent across small tubes. The change in behavior around $500 \mathrm{~km}$ corresponds to the fact that this is near the characteristic lengthscale of the $f$ mode $\left(g / \omega^{2}\right)$ of $771 \mathrm{~km}$ for the $3 \mathrm{mHz}$ centered wave packet. For larger tubes, higher- $m$ modes begin to dominate because there is little coherence of the incoming wave across the tube.

\section{Mode Conversion}

As in Cally and Bogdan (1997), we find that the magnetic flux tubes convert some of the incoming waves into slow-magnetoacoustic modes which propagate along the field-lines. This can be seen in Figure 8, where the horizontal displacement of the flux with respect to the background is shown. The vertical oscillations shown here are one signature of downward propagating slow mode waves. The magnetic nature of these waves can be easily seen in Figure 10 where a component of the magnetic-field perturbation is shown. We comment that the mode conversion removes energy from the incoming waves.

\section{Conclusions}

We have carried out a series of numerical simulations of plane-wave propagation through flux tubes of different sizes. In the first part of this study, we tested the convergence in order to determine the minimum-sized tube that we could reliably simulate with the resolutions that we had available. We then investigated how different-sized tubes interacted with an incoming $f$-mode wavepacket. Different scattered wave-field patterns were observed for flux tubes with different radii. In agreement with previous suggestions, we found that when the flux tube was small compared with the wavelength of the incoming wave, mainly the $m=1$ 
kink modes were excited. For mid-ranged tubes both the $m=1$ kink and $m=0$ sausage modes were excited, and for large tubes numerous $m$ and radial order modes were excited. Our results demonstrate that numerical simulations are an efficient, robust, and straightforward method to treat the action of flux tubes on waves regardless of their size. The simulations are a rich source of information, which can be used to better understand the observations in future studies.

\section{References}

Bogdan, T.J., Knölker, M.: 1989, Astrophys. J. 339, 579.

Bogdan, T.J., Hindman, B.W., Cally, P.S., Charbonneau, P.: 1996, Astrophys. J. 465, 406.

Cally, P.S., Bogdan, T.J.: 1997, Astrophys. J. 486, 67.

Cameron, R., Gizon, L., Daiffallah, K.: 2007, Astron. Nachr. 328, 313.

Cameron, R., Gizon, L., Duvall, T.L.: 2008, Solar Phys. 51.

Duvall, T.L., Gizon, L., Birch, A.C.: 2006, Astrophys. J. 646, 553.

Fujimura, D., Tsuneta, S.: 2009, Astrophys. J. 702, 1443-1457. doi $10.1088 / 0004-637 X / 702 / 2 / 1443$

Gizon, L., Birch, A.C., Spruit, H.C.: 2010, ArXiv:1001.0930.

Gizon, L., Hanasoge, S.M., Birch, A.C.: 2006, Astrophys. J. 643, 549. doi: $10.1086 / 502623$.

Hanasoge, S.M.: 2008, Astrophys. J. 680, 1457.

Hanasoge, S.M., Cally, P.S.: 2009, Astrophys. J. 697, 651. doi: $10.1088 / 0004-637 X / 697 / 1 / 651$.

Hanasoge, S.M., Birch, A.C., Bogdan, T.J., Gizon, L.: 2008, Astrophys. J. 680, 774. doi: $10.1086 / 587455$

Hasan, S.S., Kalkofen, L.: 1999, Astrophys. J. 519, 899.

Jain, R., Gordovskyy, M.: 2008, Solar Phys. 251, 361.

Khomenko, E., Collados, M., Fellipe, T.: 2008, Solar Phys. 251, 589.

Khomenko, E., Kosovichev, A., Collados, M., Parchevsky, K., Olshevsky, V.: 2009, Astrophys. J. 694, 411.

Roberts, B., Webb, A.R.: 1979, Solar Phys. 64, 77.

Solanki, S.K.: 1993, Space Sci. Rev. 63, 1. 
Solanki, S.K., Inhester, B., Schüssler, M.: 2006, Rep. Prog. Phys. 69, 563.

Spruit, H.C.: 1981, Astron. Astrophys. 98, 155.

Spruit, H.C.: 1982, Solar Phys. 75, 3.

Tirry, W.J.: 2000, Astrophys. J. 528, 493.

Wilson, P.R.: 1980, Astrophys. J. 237, 1008. doi:10.1086/157946. 